\title{
Early Intensive Behavioral Intervention: Outcomes for Children with Autism (Autism Spectrum Disorder)
}

\author{
Thyagarajan. R. ${ }^{1}$, Tripathi, M. A. ${ }^{1^{*}}$
}

\section{ABSTRACT}

Autism is a Neuro-developmental disability characterized by severe social, communicative and cognitive deficits, resulting in significant lifelong disability. Autism requires long-term treatment, yet, despite the severity of this disorder, some children achieve remarkable long lasting gains. Over the years, many studies have been published on comprehensive treatment approaches that seek to reduce the general level of impairment in autism. An increasing body of empirical research suggests that early, intensive, structured intervention, based on the principles of applied behavior analysis, is effective in remediating the intellectual, linguistic, and adaptive deficits associated with autism. Early intervention programs are indeed beneficial for children with autism, often improving developmental functioning and decreasing maladaptive behaviors and symptom severity at the level of group analysis. To study the efficacy of early intensive behavioral intervention, 40 children were selected and two groups were formed. Written consent was taken from parents. Therapy was provided at the centre for autism spectrum disorder, Sweekaar Academy of Rehabilitation. Individual intervention and pre-post design was used for the study. Each group consisting 20 children diagnosed with autism between age group of 3 to 6 years. Group-I received intensive behavioral intervention 4 hours 6 days a week for 1year and Group-II received regular treatment for the same period. Pre-post assessment was done by VSMS, DST, GDT, and ISSA. Result indicates significant improvement in the domain of Social Relationship and Reciprocity (SRR), Emotional Responsiveness (ER), Speech Language and Communication (L\&C), Behavior Patterns (BP), Cognitive Components (CC) Sensory Aspects (SA) and intellectual ability.

Keywords: Autism, Intensive Behavioral Intervention, Applied Behavior Analysis.

Autism is a Neuro-developmental disability characterized by severe social, communicative and cognitive deficits, resulting in significant lifelong disability. Autism requires long-term treatment, yet, despite the severity of this disorder, some children achieve remarkable long lasting gains. Over the years, many studies have been published on comprehensive treatment

\footnotetext{
${ }^{1}$ Lecturer, Institute of Mental Health, Sweekaar Academy of Rehabilitation Sciences

*Responding Author

(C) 2016 I Thyagarajan. R, Tripathi, M; licensee IJIP. This is an Open Access Research distributed under the terms of the Creative Commons Attribution License (http://creativecommons.org/licenses/by/2.0), which permits unrestricted use, distribution, and reproduction in any Medium, provided the original work is properly cited.
} 


\section{Early Intensive Behavioral Intervention: Outcomes for Children with Autism}

(Autism Spectrum Disorder)

approaches that seek to reduce the general level of impairment in autism (Dawson \& Osterling, 1997; Kasari, 2002; Rogers, 1998; Smith, 1999; Wolery \& Garfinkle, 2002). These studies highlight the importance of early comprehensive intervention services, and the fact that it is critical that children be diagnosed as early as possible and referred to appropriate effective intervention services. However, research is far from unanimous regarding the type, philosophy, and intensity of treatment that would yield valuable developmental changes. The main intervention philosophies used in intervention programs for children with autism include the developmental approach, the developmental individual-difference relationship (DIR), the treatment and education of autistic and related communication handicapped children (TEACCH), and applied behavioral analysis (ABA).

Among the important components of the intervention are teaching imitation and developing awareness of social interactions and reciprocity. This model is interdisciplinary involving speech and language, psychology, occupational, and special education therapists. Family consultation is a major component of this program (Dawson \& Osterling, 1997; Jocelyn, Casiro, Beattle, Bow, \& Kneisz, 1998; Rogers \& DiLlla, 1991).

\section{APPLIED BEHAVIOR ANALYSIS (ABA)}

Applied Behavioral Analysis (ABA) is based on scientific principles of behavior (Skinner, 1979) and is targeted at ameliorating the core deficits in autism such as communication and social skills. ABA begins with focusing on teaching small measurable units of behavior using discrete trial treatment (DTT) in mass trials. The treatment is based on systematic, step-by-step teaching using prompts and useful reinforcements.

Intervention was provided for $30 \mathrm{hrs}$ a week in one-on-one setting by experienced behavioral therapists. Each child had a separate treatment plan addressing various developmental fields, such as imitation, receptive and expressive language, joint attention, non-verbal communication, pre-academic skills, play, fine motor skills, and adaptive daily living skills. Speech and occupational therapists consulted the professional team. The program included regular preschool activities and routines such as circle time, breakfast and lunch together and play-dates. In each field goals were set according to the child's abilities, and each goal was divided into units, which were taught as separate tasks. Success in a task was defined as accurate performance in $80 \%$ of the trials based on ABA protocols (Morris, Maurice, Green, \& Luce, 1996). Intervention programs were updated weekly according to daily documented data provided by the therapists. The therapists were supervised by the psychologist who designed the child's individual treatment program. The preschool special education teacher was a senior behavioral therapist who supervised the field therapists and the implementation of the routine preschool activities.

Children are taught skills including attention, basic discrimination, language and communication, daily living, socialization, play, fine and gross motor control and pre-academics. ABA is also 


\section{Early Intensive Behavioral Intervention: Outcomes for Children with Autism}

(Autism Spectrum Disorder)

implemented in relatively unstructured situations, using incidental teaching techniques, to enhance generalization, increase motivation, and to develop social skills (Bondy \& Frost, 1994; Eikeseth, Smith, Jahr, \& Eldevik, 2002; Harris, Handleman, Gordon, Kristoff, \& Fuentes, 1991; Koegel \& Koegel, 1995; Strain, Kohler, \& Goldstein, 1996; Thorp, Stahmer, \& Schreibman, 1995). The effectiveness of these various models is a major question for the scientific community that treats children with autism. Several studies compared the effect of different treatment approaches on outcome of children with autism. Only a few studies had a control group, used standardized tests and had reliable outcome measures (Kasari, 2002). Most of the studies compared behavioral treatment programs to interventions such as "eclectic" programs (Eikeseth et al., 2002; Howard et al., 2005), school based intervention (Sheinkopf \& Siegel, 1998), parent training (Smith, Groen, \& Wynn, 2000), non-specified interventions (Birnbrauer \& Leach, 1993) and no treatment (Lovaas, 1987). Most studies reported behavioral intervention to be a more effective treatment approach (Birnbrauer \& Leach, 1993; Eikeseth et al., 2002; Howard et al., 2005; Lovaas, 1987; Sheinkopf \& Siegel, 1998; Smith et al., 2000).

The current study was conducted to compare the impact of proper structured intervention approach $\mathrm{ABA}$ and regular intervention approach. In regular intervention home based management was provided. Both programs differed in the type of professional involvement and strategies used to accomplish progress.

\section{METHODOLOGY}

\section{Aim of the Study:}

To investigate the effectiveness of early intensive behavioral intervention in autistic children

Hypothesis:

Null Hypothesis was examined with respect to intervention between both the groups

\section{Research Design:}

Individual intervention and pre-post research intervention design was used for the study. Purposive sampling was used and 40 children were selected who were diagnosed with autism according to ICD-10 criteria. Each group consists of 20 children, between age group of 3 to 6 years. Group-I received intensive behavioral intervention, 5 hours 6 days a week for 1year and Group-II parents were provided home based management, as they were from rural background, had financial constraints and non-cooperative family support, hence they could not participate in the study. Pre-post assessment was done by using VSMS, DST, GDT, and ISAA by a clinically certified Psychologist.

\section{Inclusion Criteria:}

Diagnosis of autism spectrum disorder (ICD 10), age between 3 years to 6 years, boys and girls, parents who were willing to participate 


\section{Early Intensive Behavioral Intervention: Outcomes for Children with Autism}

(Autism Spectrum Disorder)

\section{Exclusion Criteria:}

Children below 3 years and above the age of 6 years, any severe medical conditions or hearing/ visual disability

\section{Tools:}

1. Socio demographic data sheet: A socio demographic data sheet was developed and employed in the current study to collect information on relevant variables such as age, gender, religion, domicile, duration of diagnosis etc.

2. Developmental Screening Test: DST was developed by Dr. Bharath Raj in the year 1977 and was revised in 1983. The test assesses developmental skills across the child's developmental milestones which involve motor skills, language skills, mental development, self-help skills, social and emotional development from birth to 15 years of age. The test items are generally administered and scored based on the information obtained from the child's parents (essentially mother) and partly through observing the child during the clinical interview.

3. Vineland Social Maturity Scale: (VSMS) (Doll, E. A. 1953) scale was developed in 1935, at Training school, Vineland, New Jersey, USA. Its usefulness is proved in assistance with child guidance and training by indicating the relative aspects of social competence. All domains were used such as Self Help General, Self Help Dressing, Self Help Eating, Occupation, Communication, Locomotion, Self Direction, Socialization.

4. Indian Scale for Autism Assessment (ISAA): (Arya, S. 2008). It is an objective assessment tool for person with autism which uses observation, clinical evaluation of behaviour, testing by interaction with the subject and also information supplemented by parents or caretakers in order to diagnose autism. Consist 40 items rated on a 5 point scale ranging from 1 (never) to 5(always). The 40 items are divided under 6 domains, measuring the characteristic of impairments in social relationship communication and behaviour patterns of persons with autism. Scale was standardized on Indian children and having satisfactory validity and reliability

\section{Procedure:}

The study was conducted at "Sweekaar Academy of Rehabilitation Sciences" Centre for Autism Spectrum Disorders (CASD). Permission was taken from relative departments as well as management authority. Those willing to participate in the study were screened, purpose and objectives of the study was explained to all parents in a group meeting and inform consent was taken. Formal interview was conducted to collect the socio-demographic information therefore pre assessment was done in a one to one session from all participants parents. For the intervention of group I, 10 therapists was assigned, who were perusing their M.Phil in Clinical Psychology. One full day training was conducted to discuss the therapy process. This training was provided to maintain the uniformity in the therapeutic process. For group II, home based management was provided by the researchers, consisting 1hour training for three days in which 


\section{Early Intensive Behavioral Intervention: Outcomes for Children with Autism \\ (Autism Spectrum Disorder)}

target was on communication skills and they were provided the knowledge regarding how to manage their autistic children at home.

\section{INTERVENTION}

Group-I: ABA techniques-discrete trail training (DTT): The Training involves breaking behaviors into the smallest functional units and presenting them in a series. During the training process children were rewarded when adopted behaviors, and not rewarded and corrected when adopted inappropriate behaviors. In Each Discrete Trial 5 parts were used

1. Discriminative Stimulus (DS): The therapist presented a clear and small instruction such as get this, where is it, what is it etc

2. Prompt: Immediately after the DS, the therapist assisted the child to respond in a correct manner to the DS.

3. Response: Child's correct or incorrect response to the DS

4. Consequence: Correct responses were rewarded and therapist reinforces the child with hugs, claps or pat and access to his favourite toy or game. While incorrect responses were corrected.

5. Inter-trial Interval: The therapist pauses briefly for few seconds before presenting the next DS.

$$
\begin{gathered}
\text { Stimulus } \rightarrow \text { Response } \rightarrow \text { Consequence } \\
\text { OR }
\end{gathered}
$$

The trials used to develop skills, which includes domains cognitive, verbal communication, play, social and self-help skills.

\section{Domain: Cognitive skills}

One case example (child's name changed)

Concept: Throwing a ball over head Instructions in Steps

1. Therapist: "Jai, pick up the ball”.

2. The therapist takes Jai's both hands and wraps it around the ball.

3. After a short interval, the therapist says "Jai, pick up the ball” again.

4. Jai does not respond, so the therapist wraps Jai's hands around the ball again.

5. Then next, Jai makes a tentative move for the ball but the therapist still needs to wrap his/her hands around the ball. "That's a good try, Jai." (reinforce)

6. Now therapist models the act of throwing the ball over head towards Jai

7. Next therapist asks Jai to imitate the same

8. Jai does not respond, so therapist repeats the same act

9. Then Therapist helps Jai by holding his hands to throw the ball over head.

10. Jai grabs the ball and throws over head. Therapist says "That's really good, Jai!" He gives Jai a chocolate (reward). 


\section{Early Intensive Behavioral Intervention: Outcomes for Children with Autism}

(Autism Spectrum Disorder)

\section{Domain: Communication Skills}

\section{Concept: Identifying and expressing body parts}

\section{Instructions in Steps}

1. Therapist showed picture of body parts

2. Therapist by pointing ears tells loudly "ear", pointing eyes tells "eyes".

3. The therapist asks the child to identify where eye is?

4. Now the therapist asks the child to repeat "eye" with the therapist by pointing it.

5. Then the therapist shows the "eye" and asks the child to repeat what is that.

6. Then the therapist shows the "eye" and asks the child to repeat what is that.

\section{Domain: Self Help Skills}

\section{Concept: Wearing a Shirt}

\section{Instructions in Steps}

Therapist models the whole process of taking, holding, wearing and buttoning the shirt step by step.

Therapist asks Ram to take the shirt and hold it

Ram resists, the therapist then takes the shirt and gives it into his hands to hold it.

Therapist now asks Ram to stretch one hand and insert into the shirt but Ram could not do it, so therapist helps him in doing so (both hands one after the other)

Now therapist asks Ram to button the shirt

While Ram had difficulty buttoning, Therapist helps him to button the shirt.

\section{Domain: Social Skills}

\section{Concept: Interaction with peer group through play therapy}

\section{Instructions in Steps}

1. All 20 children were divided into 7 sub groups each consisting of 3 children ( $7^{\text {th }}$ group with 2 children). Each sub group was made to interact and play with different play groups, which in turn helped them to know more number of children and improve their social skills.

2. In the play therapy session, the child was placed in peer group and was made to play with other children by lending, borrowing and sharing the toys some other skills were incorporated, i.e requesting). Play therapy was conducted with different groups interchanging every week which helped the children to interact and socialize with their fellow peer group and know about each other. 


\section{Early Intensive Behavioral Intervention: Outcomes for Children with Autism \\ (Autism Spectrum Disorder)}

\section{Domain: Problem Behavior}

\section{Concept: Talking to self}

1. Whenever child talks to self, she/he is distracted by calling the name and then engaged in an activity (reinforced by any activity of child’s choice) and made to concentrate on that.

2. Child interest of activity was painting, so he was diverted to activities of painting and then once done reinforced by clapping hands or saying well done

Each child had a separate treatment plan addressing various developmental fields, such as imitation, receptive and expressive language, joint attention, non-verbal communication, preacademic skills, play, fine motor skills, and adaptive daily living skills. Speech and occupational therapists consulted the professional team. The program included regular preschool activities and routines such as circle time, breakfast and lunch together and play-dates. In addition, supervised inclusion program in a regular preschool was added for those children who had attained sufficient skills to participate and learn from typically developing children. In each field goals were set according to the child's abilities, and each goal was divided into units, which were taught as separate tasks. Success in a task was defined as accurate performance in $80 \%$ of the samples with ABA protocols (Morris, Maurice, Green, \& Luce, 1996). Intervention programs were updated weekly according to daily documented data provided by the therapists. The therapists were supervised by a trained behavior analyst who designed the child's individual treatment program.

Parent's group meeting was held once a week, supervised by a social worker and by a clinical psychologist. They were taught how to play with their children and how to address various challenging behaviors. In addition, a supervised inclusion program in a regular preschool was added for those children who had attained sufficient skills to participate and team from typically developing children. 


\section{RESULT \& DISCUSSION}

\begin{tabular}{|c|c|c|c|c|}
\hline \multirow[b]{2}{*}{ Age } & \multicolumn{3}{|c|}{3 to 6} & \\
\hline & \multicolumn{2}{|c|}{ Group I } & Group II & \\
\hline \multirow[t]{2}{*}{ Sex } & Male & 11 & 16 & \multirow[b]{2}{*}{40} \\
\hline & Female & 9 & 4 & \\
\hline \multirow[t]{3}{*}{ Religion } & Hindu & 11 & 13 & \multirow[b]{3}{*}{40} \\
\hline & Muslim & 5 & 5 & \\
\hline & Christian & 4 & 2 & \\
\hline \multirow[t]{2}{*}{ Family type } & Joint & 2 & 12 & \multirow[b]{2}{*}{40} \\
\hline & Nuclear & 18 & 8 & \\
\hline \multirow[t]{2}{*}{ Habitat } & Urban & 20 & 0 & \multirow[b]{2}{*}{40} \\
\hline & Rural & 0 & 20 & \\
\hline \multirow[t]{2}{*}{ Family Income } & $<$ Rs10,000/- & 0 & 20 & \multirow[b]{2}{*}{40} \\
\hline & >Rs. 10,000/- & 20 & 0 & \\
\hline \multirow[t]{3}{*}{ Parent's Education } & Illiterate & 0 & 8 & \multirow{3}{*}{40} \\
\hline & $<10^{\text {th }}$ & & 12 & \\
\hline & $>10^{\text {th }}$ & 20 & & \\
\hline
\end{tabular}

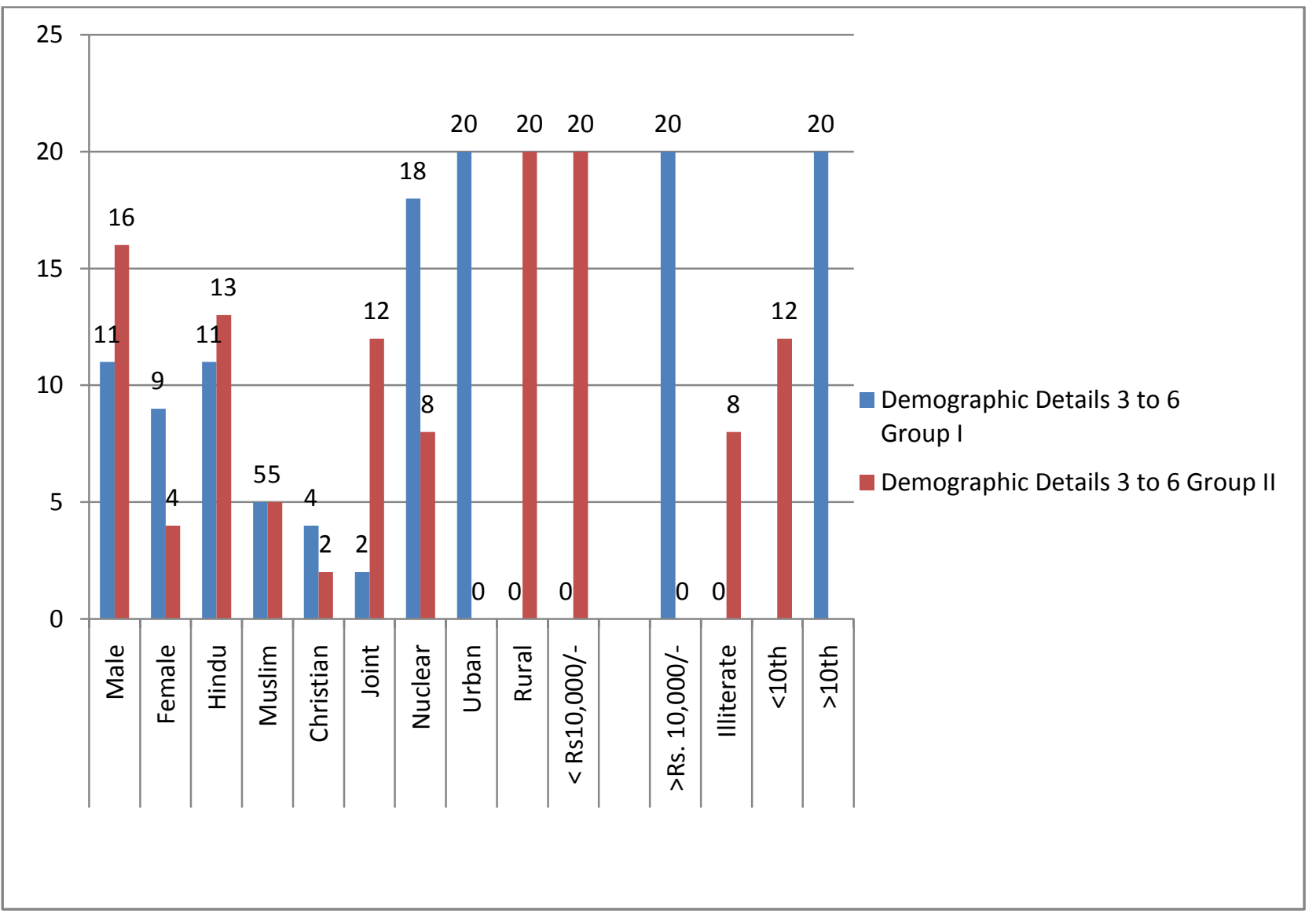




\section{Early Intensive Behavioral Intervention: Outcomes for Children with Autism \\ (Autism Spectrum Disorder)}

Table 2 graphical representation indicates there are more number of males in both the groups when compared to female, Hindus are more when compared to other religion, subjects from joint family are more in group II than group I, when compared to group I most of the subjects are from nuclear family hailing from Urban area with literacy rate more than $10^{\text {th }}$ standard and higher socio economic status than group II.

Table: 2 Mean (SD) scores of ISAA in Group I and Group II pre and post intervention

\begin{tabular}{|c|c|c|c|c|}
\hline \multirow[t]{2}{*}{ Group } & \multicolumn{2}{|c|}{ ISAA } & \multirow{2}{*}{$\mathrm{t}$} & \multirow[b]{2}{*}{$\mathbf{P}$} \\
\hline & Pre-Test & Post Test & & \\
\hline Group I & $\begin{array}{l}\text { Mean } \\
\text { (SD) }\end{array}$ & Mean (SD) & \multirow{3}{*}{11.7} & \\
\hline & $\begin{array}{l}128.15 \\
(16.6)\end{array}$ & $\begin{array}{l}86.25 \\
(9.98)\end{array}$ & & .000 \\
\hline Group II & $\begin{array}{l}129.35 \\
(16.5)\end{array}$ & $\begin{array}{l}130.95 \\
(17.8)\end{array}$ & & .619 \\
\hline
\end{tabular}

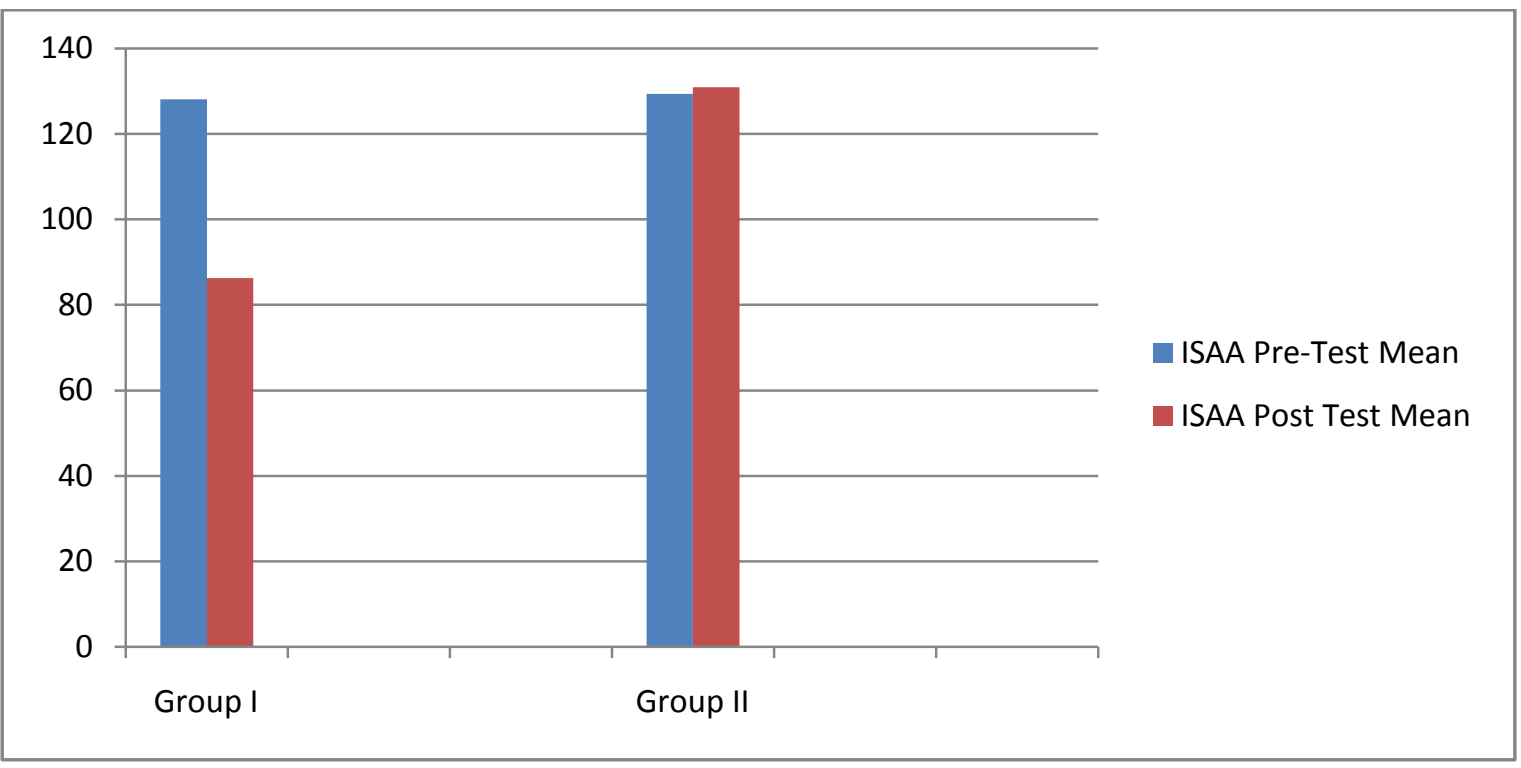

There is significant difference on ISAA scores in Group I when compared to Group II, in all domains group I improved more than the group II. Paired comparisons tests for each group separately showed that the pre- and post intervention differences were significant for group I. 
Early Intensive Behavioral Intervention: Outcomes for Children with Autism (Autism Spectrum Disorder)

Table 3 Mean (SD) scores of DQ of Group I and Group II pre and post intervention

\begin{tabular}{|l|l|l|l|l|}
\hline Group & DQ & & \\
\hline & Pre-Test & Post-Test & t & p \\
\hline Group I & Mean (SD) & Mean (SD) & & \\
\hline & 63.55 & 70.7 & & \\
& $(4.1)$ & $(3.05)$ & \multirow{2}{*}{-9.4} & .000 \\
\hline Group II & 61.85 & 58.60 & & .008 \\
& $(5.69)$ & $(8.54)$ & & \\
\hline
\end{tabular}

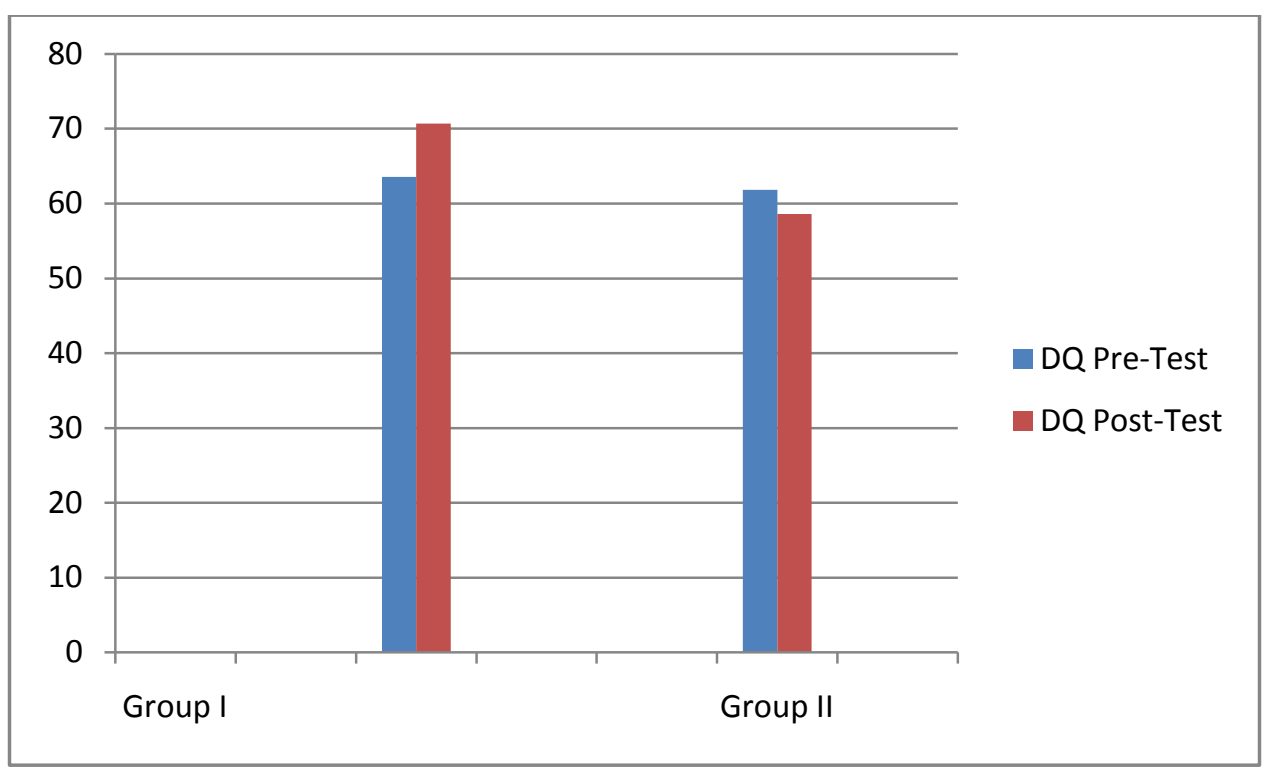

Table 4 Mean (SD) scores of SQ in Group I and Group II pre and post intervention

\begin{tabular}{|l|l|l|l|l|}
\hline & \multicolumn{2}{|c|}{ SQ } & \multirow{2}{*}{ t } & \multicolumn{2}{c|}{ p } \\
\hline \multirow{2}{*}{ Group } & Pre-Test & Post-Test & & \\
\hline & Mean (SD) & Mean (SD) & & .000 \\
\hline Group I & $\begin{array}{l}63.70 \\
(3.79)\end{array}$ & $\begin{array}{l}70.95 \\
(3.53)\end{array}$ & & \\
\hline Group II & $\begin{array}{l}63.5 \\
(6.33)\end{array}$ & $\begin{array}{l}53.9 \\
(7.28)\end{array}$ & \multirow{2}{*}{-7.1} & .001 \\
\hline
\end{tabular}


(Autism Spectrum Disorder)

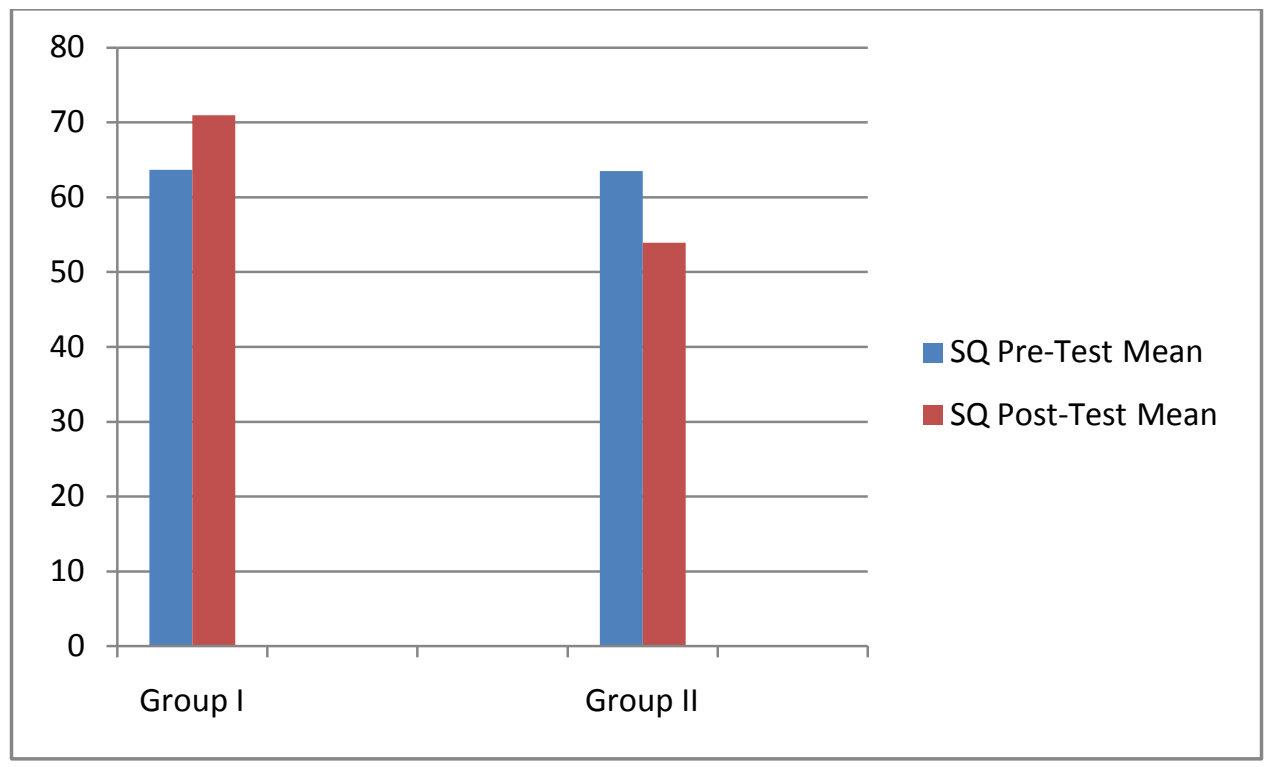

Graphical representation of table 3 and 4 explain Group I, having significantly higher post treatment DQ scores with 7 points increase, where as Group II found to be having change in the scores with 3 points decrease. In the SQ scores, there was significant difference post intervention even, as it was 7 points increase on an average in Group I and 10 points decrease in the Group II.

\section{Table 5 Group I: ISAA scores}

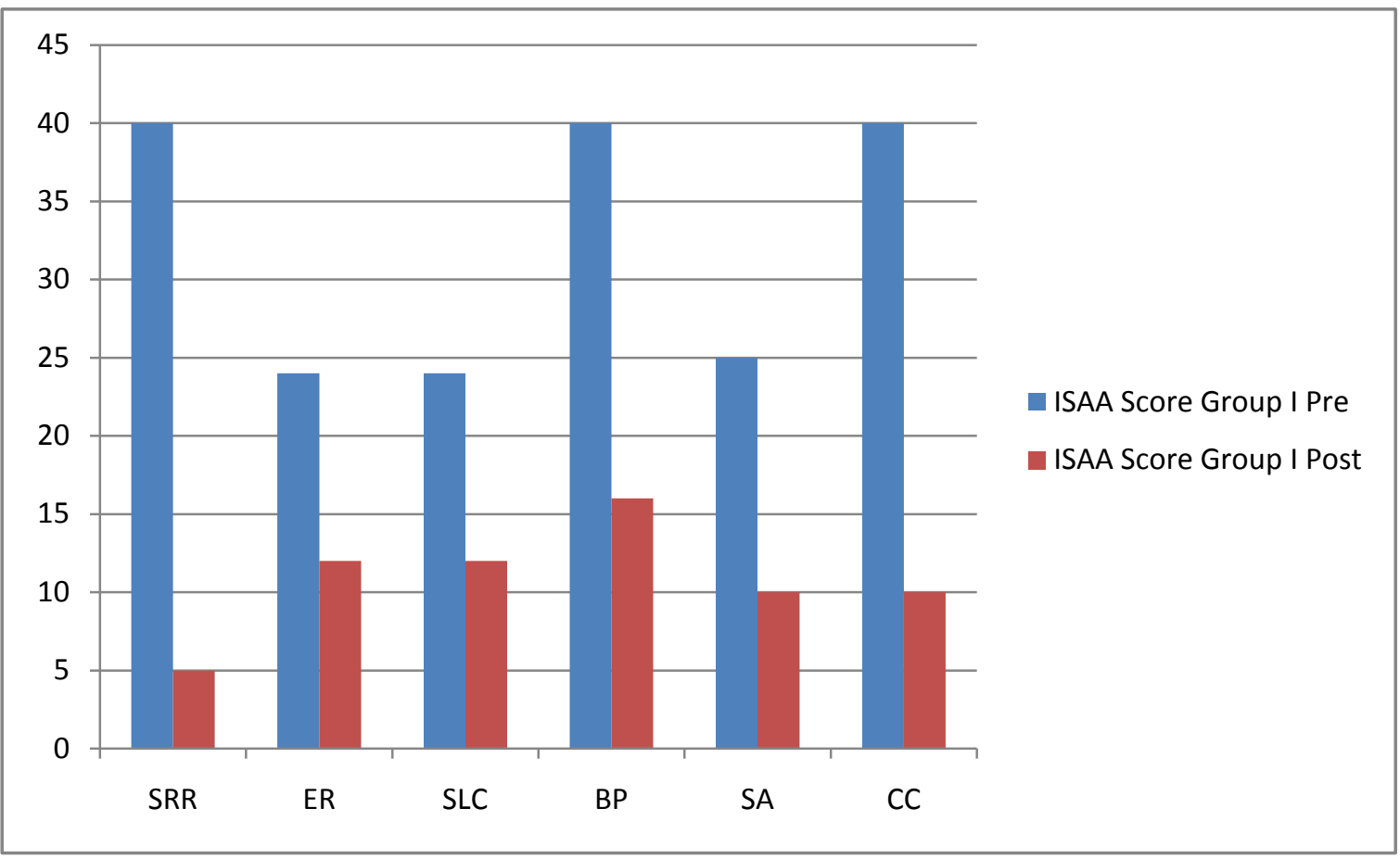




\section{Early Intensive Behavioral Intervention: Outcomes for Children with Autism}

(Autism Spectrum Disorder)

Table 6 Group II: Average ISAA Scores of 20 subjects

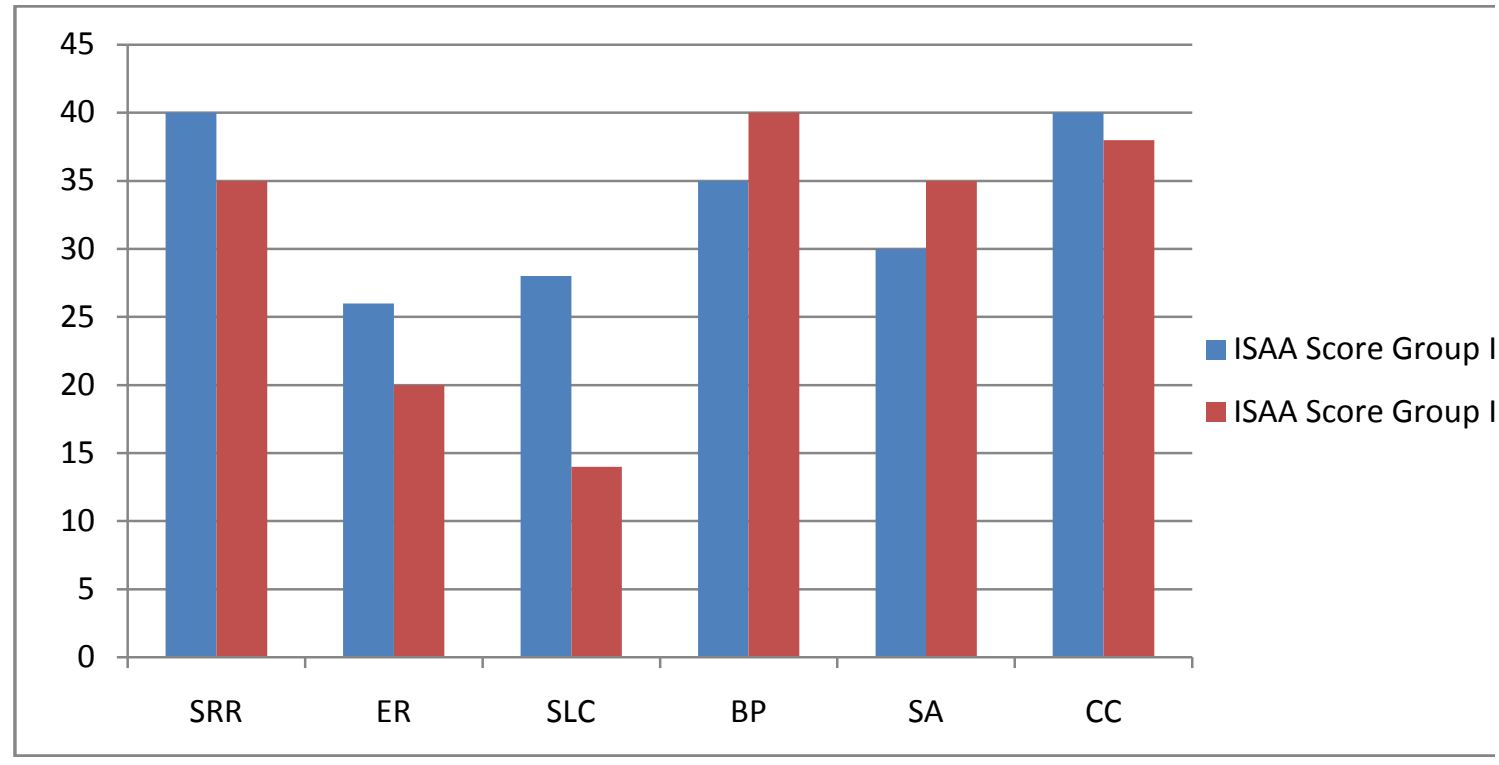

Table 5 \& 6 describes scores of Group I on ISAA pre and post intervention, on the domain of social relationship and reciprocity scored high 40 on no eye contact, post intervention there was a drastic change where eye contact was maintained by all but 5 subjects were needed prompts at times, indicating a score of 05 . On emotional response domain self stimulatory and exaggerated emotions was 24, post intervention it is reduced to 12, On speech language and communication domains such as using different non-verbal language, producing echolalic language also showed improvement in the score by frequency reduction from 24 to 12, on behavioral patterns, hyperactivity, restlessness and attention to objects, on which Group I showed a drastic change from a score of 40 to 16, On sensory aspects, there was difficulty in tracking objects, insensitive to pain , responding to objects or people unusually a score of 25 has reduced to 10 , indicating improvement in tracking objects, sensitivity to pain, On cognitive components, inattention and delay in response was common among many subjects, which showed a good improvement post therapy from a score of 40 to 10 . The same findings on all domains were not observed in the group II regular intervention group, but showed improvement in social relationship and reciprocity, emotional response, speech language and communication. After 1 year of intervention, however advancement in all domains is more pronounced in the group I, whereas changes in the group II is based on the psycho education and home based training given to the parents after being diagnosed with autism.

\section{DISCUSSION}

Discrepancies between the groups are more apparent in language and communication, as only Group I, showed significant progress. Previous studies also report that early intervention can produce significant behavioral changes (Rogers, 1998; Smith, 1999). The current study 


\section{Early Intensive Behavioral Intervention: Outcomes for Children with Autism (Autism Spectrum Disorder)}

emphasizes the importance of the type of intervention used. Most previous pre-post intervention studies in autism compare behavioral intervention to other philosophy based approaches (Birnbrauer \& Leach, 1993; Eikeseth et al., 2002; Howard et al., 2005; Sheinkopf \& Siegel, 1998; Smith et al., 2000). This study adds to previous reports by concentrating on improvement of core autistic features, while others report on progress mostly in cognitive abilities, (Birnbrauer \& Leach, 1993; Eikeseth et al., 2002; Howard et al., 2005; Lovaas, 1987; Sheinkopf \& Siegel, 1998), language and adaptive skills measures (Birnbrauer \& Leach, 1993; Eikeseth et al., 2002; Howard et al., 2005).

Possible explanation for the ABA advantage lies in the use of structured teaching setting, well defined learning goals and using simple instructions with many repetitions until the goal is achieved. Teaching attention and learning skills in the beginning of the intervention is highly important as children with autism have difficulties diverting their attention to various stimuli in the environment. Some children with autism have difficulties in learning from the natural environment therefore, unstructured, flexible and incidental teaching program appears less suitable for them.

ABA differs also in the teaching format from other programs. Teaching basic skills in one-onone setting is perhaps superior to teaching in group format. In addition, social reward plays an important role in the learning of typically developing children. Unfortunately, children with autism do not always respond to these types of social rewards. Therefore, using an individualized reward system that is derived from the child's preferences, as used in ABA, seems to be a crucial factor in modifying behavior patterns and progress in learning.

In $\mathrm{ABA}$, teaching is more consistent, both in the methods used and in the physical environment and changes are made gradually according to the child's progress. ABA is based on established protocols and therefore is applied more consistently and is less affected by the differences between the individual therapists.

Several studies use DQ as an independent and an outcome measure at the same time which adds a level of bias toward positive outcome (Matson, 2006). In the current study DQ is used only as the independent measure. Children in the group I improved more than children in the group II, regardless of their baseline DQ level. This finding points to the advantage of ABA intervention for a range of cognitive abilities in children with autism. In this study, diagnosis of autism of all the participants is overall quite stable with $90 \%$ remaining within the autism/ASD categories.

To date, the majority of outcome studies do not use one of the primary measures of autism as an outcome measure (Rogers, 1998). A minority of studies that look at reduction of autism symptoms used the autism behavior checklist (ABC) (Krug, Arick, \& Almond, 1980), or the childhood autism rating scales (CARS) (Jocelyn et al., 1998; Ozonoff \& Cathcart, 1998) which 


\section{Early Intensive Behavioral Intervention: Outcomes for Children with Autism (Autism Spectrum Disorder)}

are not based on DSM-IV criteria. The current study has stringent matching criteria in reference to the children's age, profile and intensity of intervention in both groups. Prior to intervention, children in both comparison groups are not significantly different in their global diagnosis of autism or in their autism severity in language and communication, and in reciprocal social domains.

The current study shows that very young children with autism improve significantly with early intervention. However, the type of intervention applied has a major impact on this progress. Change in core autism symptoms is more apparent with intervention based on ABA principles in comparison to group II. Future research may also look at the profile of children who gain more from a specific intervention and investigate the long-term outcome of ABA. This may help parents and therapists to choose the intervention program for each child.

\section{LIMITATIONS}

Group II is not properly controlled, ABA intervention to the learners made meaningful changes in many areas as per the need. However, changes did not typically occur quickly. Rather, most learners require intensive and ongoing instruction that builds their progress step-by-step. Moreover, the rate of progress-like the goals of intervention-varies considerably from person to person depending on age, level of functioning, family goals and other factors

\section{REFERENCES}

American Psychiatric Association (APA) (1994). Diagnostic and statistical manual of mental disorders (4th Ed.), Washington, DC.

Birnbrauer, J. S., \& Leach, D. J. (1993). The Murdoch early intervention program after 2 years. Behavior Change, 10, 63-74.

Birnbrauer, S. J., \& Leach, J. D. (1993). The murdoch early intervention program after 2 years, department of psychology; Murdoch University, 10, 02, 63-74

Bondy, A. S., \& Frost, L. A. (1994). The picture exchange communication system. Focus on Autistic Behavior, 9, 1-19.

Christina M. Corsello (2005), Early Intervention in Autism, Infants \& Young Children Vol. 18, No. 2, pp. 74-85., Lippincott Williams \& Wilkins.

Dawson, G., \& Osterling, J. (1997). Early intervention in autism. In M. J. Guralnick (Ed.), The effectiveness of early intervention (pp. 307-326). Baltimore, MD: Paul H. Brookes

E. Hibbs \& P. Jensen (Eds.), Psychosocial treatments for child and adolescent disorders: Empirically based strategies for clinical practice (pp. 573-586).

Eikeseth, S., Smith, T., Jahr, E.,\&Eldevik, S. (2002). Intensive behavioral treatment at school for 4- to 7-year-old children with autism. Behavior Modification, 26, 49-68.

Harris, S. L., Handleman, J. S., Gordon, R., Kristoff, B., \& Fuentes, F. (1991). Changes in cognitive and language functioning of preschool children with autism. Journal of Autism and Developmental Disorders, 21, 281-290. 


\section{Early Intensive Behavioral Intervention: Outcomes for Children with Autism \\ (Autism Spectrum Disorder)}

Howard, J. S., Sparkman, C. R., Cohen, H. G., Green, G., \& Stanislaw, H. (2005). A comparison of intensive behavior analytic and eclectic treatments for young children with autism. Research in Developmental Disabilities, 26, 359-383.

Howard, S. j., Sparkman, R. C., Cohen, G. H., et al. (2005). A comparison of intensive behavior analytic and eclectic treatments of young children with autism. Research in Developmental Disabilities, 359-383.

Jocelyn, L. J., Casiro, O. G., Beattle, D., Bow, J., \& Kneisz, J. (1998). Treatment of children with autism: A randomized controlled trial to evaluate a caregiver-based intervention program in community day-care centers. Journal of Developmental and Behavioral Pediatrics, 19, 326-334.

Kasari, K. (2002). Assessing changes in early intervention programs for children with autism. Journal of Autism and Developmental Disorders, 32, 447-461.

Koegel, R. L., \& Koegel, L. K. (1995). Teaching children with autism. Baltimore: Paul H Brookes.

Krug, D. A., Arick, J., Almond, P. (1980). Behavior checklist for identifying severely handicapped individuals with high levels of autistic behavior. Journal of Child Psychology Psychiatry, 21 (3):221-9.

Lovaas, O. I. (1987). Behavioral treatment and normal educational and intellectual functioning in young autistic children. Journal of Consulting \& Clinical Psychology, 55, 3-9.

Matson , L. J., Wilkins, J., \& Gonza'lez, M. (2008). Early identification and diagnosis in autism spectrum disorders in young children and infants: How early is too early? Research in Autism Spectrum Disorders ( $2^{\text {nd }}$ Ed) $75-84$

Morris, J. L., Maurice, C., Greene, G., \& Luce, S. C. (1996). Behavioral intervention for young children with autism.

Oosterling, I., Visser, J., Swinkels, S. et al. (2010), Randomized controlled trial of the focus parent training for toddlers with autism. Journal of Autism Developmental Disorder 40(12): 1447-1458.

Ozonoff, S., \& Cathcart, K. (1998). Effectiveness of a home program intervention for young children with autism. Journal of Autism and Developmental Disorders, 28(1), 25-32.

Rogers, S. J. (1998). Empirically supported comprehensive treatments for young children with autism. Journal of Clinical Psychology, 21, 168-179.

Rogers, S. J., \& DiLlla, D. I. (1991). A comparative study of the effects of develop mentally based instructional model on young children with other disorders of behavior and development. Topics in Early Childhood Special Education, 77, 29-47.

Rogers, S. J., \& Vismara, A. L. (2007). Evidence based psychosocial treatments of child and adolescent disorders. In press, Journal of Clinical Child and Adolescent Psychology for Special Issue on M.I.N.D. Institute University of California Davis.

Schreibman, L., \& Ingersoll, B. (2005). Behavioral interventions to promote learning in individuals with autism. F. Volkmar, R. Paul, A. Klin, R. \& D. Cohen (Eds.). Handbook 


\section{Early Intensive Behavioral Intervention: Outcomes for Children with Autism \\ (Autism Spectrum Disorder)}

of autism and pervasive developmental disorders ( $3^{\text {rd }}$ Eds). Pp- 882-896. Hoboken, NJ: John Wiley \& Sons, Inc.

Sheinkopf, S. J., \& Siegel, B. (1998). Home based behavioral treatment of young autistic children. Journal of Autism and Developmental Disorders, 28, 15-24.

Skinner, N. F. (1979). Learned helplessness performance as a function task significance. Journal of Psychology, 102,77-82.

Smith, T. (1999). Outcome of early intervention for children with autism. Clinical Psychology: Science and Practice, 6, 33-48.

Smith, T., Groen, A. D., \& Wynn, J. W. (2000). Randomized trail of intensive early intervention for children with pervasive developmental disorder. American Journal of Mental Retardation, 105, 269-285.

Strain, P. S., Kohler, F. W., \& Goldstein, H. (1996). Learning experiences: An alternative program: Peer-mediated intervention for young children with autism.

Thorp, D. M., Stahmer, A. C., \& Schreibman, L. (1995). Effects of socio-dramatic play training on children with autism. Journal of Autism and Developmental Disorders, 25, 265-282.

Wolery, M., \& Garfinkle, A. N. (2002). Measures in intervention research with young children who have autism. Journal of Autism and Developmental Diorders, 32, 463-478. 